view this as a philosophical rather than a scientific issue. Certainly, the unusual behaviour of the Clcn4 gene in the mouse does add a small but important piece of information to our knowledge of sex chromosome evolution in mammals and should be considered as just another brick in the wall. Models suchas the one proposed by Jennifer Marshall Graves for the evolution of the murine pseudo- autosomal region, although appealing, represent only one of several possible scenarios with both supporting and contradicting evidence. More data need to be obtained on the pseudoautosomal region in several mammalian species, including the mouse, before we can reasonably guess what happened to our sex chromosomes during the past few million years.

\section{Andrea Ballabio}

Telethon Institute of Genetics and Medicine (TIGEM), San Raffaele Biomedical Science Park, Via Olgettina 58, 20132 Milan, Italy

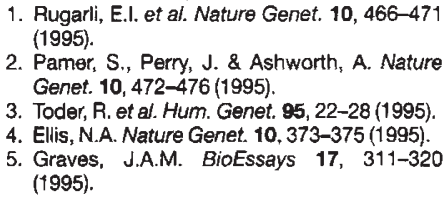
(1995).

2. Pamer, S., Perry, J. \& Ashworth, A. Nature Genet. 10, 472-476 (1995)

3. Toder, R. et al. Hum. Genet. 95, 22-28 (1995).

4. Ellis, N.A. Nature Genet. 10,373-375 (1995).

5. Graves, J.A.M. BioEssays 17, 311-320 (1995).

\title{
Glucagon receptor gene mutation in essential hypertension
}

Sir - A missense mutation in the glucagon receptor (GCG-R) gene (Gly40Ser) was recently described in $5 \%$ of French patients with late onset (48 \pm 13 SD years) noninsulin-dependent diabetes mellitus (NIDDM), but was found in only $1 \%$ of healthy controls ${ }^{1}$. A role in NIDDM, perhaps involving a disturbance in the insulinotropic effect of glucagon on pancreatic $\beta$-cells, was therefore invoked ${ }^{1}$. This finding has been confirmed in a study of three regional groups of NIDDM patients in the UK (frequency $=2 \%$ in NIDDM cf. $0.2 \%$ in controls), with a similar prevalence also being noted in patients with insulin-dependent diabetes mellitus (IDDM) ${ }^{2}$.

Since mild glucose intolerance is seen in essential hypertension (HT) and has been implicated in the genetics of HT aetiology $y^{3-5}$, we examined the GCG-R Gly40Ser status of a well-studied cohort of 130 nondiabetic Caucasian patients with a strong family history of HT and a control group of 90 subjects whose parents were both normotensive past the age of 50 years $^{6}$. Leukocyte DNA was genotyped for the mutation by PCR and BstEII digestion as described ${ }^{1}$.

Heterozygosity for the Gly40Ser mutation was seen in $7(5 \%)$ of the HTs, but in only one $(1 \%)$ of controls; genotype frequencies resembled those originally reported in NIDDM cases and controls ${ }^{1}$. Moreover, HTs with the mutation displayed significantly later onset of $\mathrm{HT}$ than HTs who lacked it ( $52 \pm 5$ vs 40 \pm 2 SE years; $P<0.0001)$. Furthermore, all of the HTs with the mutation were female, whereas the single mutant control was a male ( $\chi^{2} 1$ d.f. $=6.4 ; P=0.01)$ who was aged 59 .

The present study therefore implicates the GCG-R Gly40Ser variant or a polymorphism in linkage disequilibrium with it on chromosome $17 q 25$ in disease aetiology in a subset of HT patients. It also indicates that the mutation is just as prevalent in another member of the 'Syndrome X' complex polygenic disease constellation, which includes HT, diabetes, atherosclerosis, dyslipidaemia and obesity ${ }^{4}$. Different cofactors may subsequently be required for individuals with the predisposing mutation to then go on to develop one disease or the other.

Curiously, in the IDDM study there was a lack of preferential transmission of the mutation from heterozygous parents to affected sibs ${ }^{2}$. Since both IDDM and NIDDM patients are at increased risk of developing $\mathrm{HT}$, the present findings could provide a possible explanation for such apparent discrepancies. The prevalence of HT in NIDDM can run as high as $40 \%$ in male and over $50 \%$ in female patients. This is partly explained by obesity, older age and essential HT, where the latter often precedes NIDDM and reflects the familial clustering of essential HT, NIDDM and obesity ${ }^{7}$. Moreover, renal disease is seen in $20-30 \%$ of NIDDM patients and could contribute to blood pressure increases in a proportion of patients. In IDDM, nephropathy develops in 30-40\% of cases and is more likely to occur in those with a family history of essential HT. The links between HT and diabetes are therefore ample and could impinge on the genetic findings for GCG-R.

Transfected BHK cells expressing receptors with the Gly40Ser mutation have lower affinity for glucagon $^{1}$, as well as reduced response in cAMP $^{8}$. Glucagon receptors are expressed in normal pancreatic $\beta$ cells ${ }^{9}$ and, in transfected RIN cells expressing mutant receptors, the response in insulin release to glucagon is blunted ${ }^{8}$. Although HT patients show a certain degree of hyperinsulinaemia, their insulin secretion could be regarded as inadequate, given their hyperglycaemia. Also, mutant GCG$R$ in kidney would enhnace the antinatriuretic effect of insulin, However, pathophysiological mechanisms connected with the present HT association finding can only be speculated on at this stage.

\section{Susan M. Chambers}

Brian J. Morris

Molecular Biology \& Hypertension Laboratory, Department of Physiology and The Sydney Institute for Biomedical Research (F13), The University of Sydney, NSW 2006, Australia

\footnotetext{
1. Hager, J. et ai. Nature Genet. 9, 299-304 (1995).

2. Gough, S.C.L. et al. Hum. Mol. Genet. 4 , 1609-1612 (1995).

3. Modan, M. et al. J. Clin. Invest. 75, 809-817 (1985).

4. Reaven, G.M. \& Hofiman, B.B. Am. J. Med. 87 (suppl 6A), 2-6S (1989).

5. Allemann, Y. et al. Lancet 341, 327-331 (1993)

6. Morris, B.J. et al. J. Clin. invest. 94, 1085-1089 (1994).

7. Stern, N. \& Tuck, M.L. in Hypertension: Pathophysiology, Diagnosis and Management (eds Laragh, J.H. \& Brenner, B.M.) 2301-2314 (Raven Press, New York, 1995).

8. Hansen, L. et al. Diabetes (in the press).

9. Kawai, K. et al. Diabetologia 38, 274-276 (1995).
} 